\title{
The SARS-CoV-2 Omicron (B.1.1.529) variant exhibits altered pathogenicity, transmissibility, and fitness in the golden Syrian hamster model
}

\section{Authors:}

Shuofeng Yuan ${ }^{1, \dagger}$, Zi-Wei Ye ${ }^{1, \dagger}$, Ronghui Liang ${ }^{1, \dagger}$, Kaiming Tang ${ }^{1, \dagger}$, Anna Jinxia Zhang ${ }^{1, \dagger}$, Gang $\mathrm{Lu}^{2,3, \dagger}$, Chon Phin Ong ${ }^{4, \dagger}$, Vincent Kwok-Man Poon ${ }^{1,5}$, Chris Chung-Sing Chan ${ }^{1,5}$, Bobo WingYee Mok ${ }^{1}$, Zhenzhi Qin ${ }^{1}$, Yubin Xie ${ }^{1}$, Haoran Sun ${ }^{1}$, Jessica Oi-Ling Tsang ${ }^{1,5}$, Terrence Tsz-Tai Yuen $^{1}$, Kenn Ka-Heng Chik ${ }^{1,5}$, Chris Chun-Yiu Chan ${ }^{1}$, Jian-Piao Cai ${ }^{1}$, Cuiting Luo ${ }^{1}$, Lu Lu ${ }^{1,5}$, Cyril Chik-Yan Yip ${ }^{6}$, Hin Chu ${ }^{1,3,5}$, Kelvin Kai-Wang To ${ }^{1,5,6,7,8}$, Honglin Chen ${ }^{1,5,7,8}$, Dong-Yan Jin $^{4,8, 末}$, Kwok-Yung Yuen ${ }^{1,3,5,6,7,8, \ddagger} \&$ Jasper Fuk-Woo Chan ${ }^{1,3,5,6,7,8, \dot{+}, *}$

${ }^{\dagger}$ These authors are co-first authors.

These authors are co-last authors.

\section{Affiliations:}

${ }^{1}$ State Key Laboratory of Emerging Infectious Diseases, Carol Yu Centre for Infection, Department of Microbiology, Li Ka Shing Faculty of Medicine, The University of Hong Kong, Pokfulam, Hong Kong Special Administrative Region, China.

${ }^{2}$ Key Laboratory of Tropical Translational Medicine of Ministry of Education, Hainan Medical University, Haikou, Hainan, China.

${ }^{3}$ Academician Workstation of Hainan Province, Hainan Medical University-The University of Hong Kong Joint Laboratory of Tropical Infectious Diseases, Hainan Medical University, Haikou, Hainan, China.

${ }^{4}$ School of Biomedical Sciences, Li Ka Shing Faculty of Medicine, The University of Hong Kong, Pokfulam, Hong Kong Special Administrative Region, China.

${ }^{5}$ Centre for Virology, Vaccinology and Therapeutics, Hong Kong Science and Technology Park, Hong Kong Special Administrative Region, China.

${ }^{6}$ Department of Microbiology, Queen Mary Hospital, Pokfulam, Hong Kong Special Administrative Region, China. 
$30 \quad{ }^{7}$ Department of Clinical Microbiology and Infection Control, The University of Hong Kong-

31 Shenzhen Hospital, Shenzhen, Guangdong, China.

$32{ }^{8}$ Guangzhou Laboratory, Guangzhou, Guangdong Province, China.

33

$34 *$ Address correspondence to Jasper Fuk-Woo Chan (jfwchan@hku.hk). State Key Laboratory of

35 Emerging Infectious Diseases, Carol Yu Centre for Infection, Department of Microbiology, Li

36 Ka Shing Faculty of Medicine, The University of Hong Kong, Pokfulam, Hong Kong Special

37 Administrative Region, China; and Department of Clinical Microbiology and Infection Control,

38 The University of Hong Kong-Shenzhen Hospital, Shenzhen, Guangdong, China. Tel: +852-

3922552402.

40

41 Word count: Abstract: 247; Text: 4607.

42

43 One Sentence Summary: The novel SARS-CoV-2 Omicron variant, though less pathogenic, is

44 highly transmissible and outcompetes the Delta variant under immune selection pressure in the 45 golden Syrian hamster COVID-19 model. 


\section{$47 \quad$ ABSTRACT}

48 The newly emerging SARS-CoV-2 Omicron (B.1.1.529) variant first identified in South Africa

49 in November 2021 is characterized by an unual number of amino acid mutations in its spike

50 that renders existing vaccines and therapeutic monoclonal antibodies dramatically less effective.

51 The in vivo pathogenicity, transmissibility, and fitness of this new Variant of Concerns are

52 unknown. We investigated these virological attributes of the Omicron variant in comparison with

53 those of the currently dominant Delta (B.1.617.2) variant in the golden Syrian hamster COVID-

5419 model. Omicron-infected hamsters developed significantly less body weight losses, clinical

55 scores, respiratory tract viral burdens, cytokine/chemokine dysregulation, and tissue damages

56 than Delta-infected hamsters. The Omicron and Delta variant were both highly transmissible

$57(100 \%$ vs 100\%) via contact transmission. Importantly, the Omicron variant consistently

58 demonstrated about $10-20 \%$ higher transmissibility than the already-highly transmissible Delta

59 variant in repeated non-contact transmission studies (overall: $30 / 36$ vs 24/36, 83.3\% vs 66.7\%).

60 The Delta variant displayed higher fitness advantage than the Omicron variant without selection

61 pressure in both in vitro and in vivo competition models. However, this scenario drastically

62 changed once immune selection pressure with neutralizing antibodies active against the Delta

63 variant but poorly active against the Omicron variant were introduced, with the Omicron variant

64 significantly outcompeting the Delta variant. Taken together, our findings demonstrated that

65 while the Omicron variant is less pathogenic than the Delta variant, it is highly transmissible and

66 can outcompete the Delta variant under immune selection pressure. Next-generation vaccines

67 and antivirals effective against this new VOC are urgently needed. 


\section{INTRODUCTION}

69 Severe acute respiratory syndrome coronavirus 2 (SARS-CoV-2) was first identified in late 2019 and quickly developed into the most important global health challenge in recent decades (1-3).

71 Despite rapid generation of vaccines and antivirals, and global implementation of various non-

72 pharmaceutical public health measures, the Coronavirus Disease 2019 (COVID-19) pandemic

73 continues to rage on nearly two years with the emergence of more Variants of Concern (VOCs)

74 that exhibit immune evasion and/or enhanced transmissibility (4). The Alpha (B.1.1.7) variant

75 emerged in mid-2020 and quickly outcompeted the Beta (B.1.351) variant (5,6). The currently

76 dominant Delta (B.1.617.2) variant with enhanced transmissibility and moderate level of

77 antibody resistance then replaced the Alpha (B.1.1.7) variant since mid-2021.

79 November 2021, has now affected more than 100 other countries/regions $(7,8)$. This new VOC

80 has an alarmingly high number of mutations $(>30)$ at the spike, which significantly reduce the

81 neutralizing activity of vaccine-induced serum antibodies, as well as therapeutic monoclonal

82 antibodies (9-15). Preliminary analyses of the severity of infections caused by the Omicron

83 variant compared to previous variants as determined by hospitalization rates have been

84 inconclusive, with some showing reduced hospitalization rate and others showing a lack of

85 significant difference (16). What is more apparent from early epidemiological data is that the

86 Omicron variant is spreading rapidly even in populations with high two-dose COVID-19

87 vaccination uptake rates $(17,18)$. However, whether this is due to the intrinsic transmissibility of the Omicron variant or other extrinsic environmental and social factors is unknown. At present, the in vivo pathogenicity, transmissibility, and fitness of this variant are poorly understood. In 
91 with those of the presently dominant Delta variant in the established golden Syrian hamster

92 model for COVID-19, which closely simulates non-lethal human disease (19).

\section{RESULTS}

The Omicron variant is less pathogenic than the Delta variant in vivo

We first compared the clinical signs, viral burden, and cytokine/chemokine profiles of the the Omicron-infected hamsters were limited, and consistently and significantly milder than those

(Fig. S1A) and feces (Fig. S1B) became undetectable by 12 dpi and 14 dpi, which were both earlier than the Delta variant. Corroborative to the viral burden findings, the Omicron-infected

112 hamsters had generally lower tissue cytokines/chemokines gene expression levels between 2 dpi 113 and 7 dpi (Fig. 1G). At 7 dpi, the dysregulated inflammatory cytokine/chemokine response has 
114 almost completely normalized. The antibody response against variant-specific spike receptor115 binding domain (RBD) of the Omicron-infected hamsters was also significantly lower than that 116 of the Delta-infected hamsters (Fig. S2). wall congestion, while the Delta-infected hamsters demonstrated more severe and diffuse alveolar wall infiltration and congestion (Fig. S3). At 4 dpi, both groups of hamsters showed bronchiolar epithelial destruction, and peribroncheolar and perivascular inflammatory

121 infiltration, but the alveolitis in the Delta-infected hamsters was more much more diffuse than 122 the Omicron-infected hamsters. At $7 \mathrm{dpi}$, the lung sections of the Omicron-infected hamsters 123 appeared mostly normal, while those of the Delta-infected hamsters still showed blood vessel 124 congestion and alveolar wall inflammatory infiltration, indicating that the Omicron-infected hamsters had earlier resolution of tissue damage. Viral nucleocapsid protein expression was 126 significantly more abundantly seen in the lung sections of the Delta-infected than the Omicron127 infected hamsters throughout 2 dpi to 7 dpi (Fig. S4). Similarly, the Omicron-infected hamsters showed less severe histopathological changes (Fig. S5) and less abundant viral nucleocapsid 129 protein expression (Fig. S6) in their nasal turbinate than the Delta-infected hamsters from 4 dpi to 7 dpi.

133 The other key question was the comparative transmissibility of the Omicron and Delta variants in 134 vivo. To this end, we first co-housed 6 index SARS-CoV-2-challenged hamsters ( $\mathrm{n}=3$ each for the Omicron and Delta variants) with 6 naïve hamsters for 4 hours in a 1:1 ratio (Fig. 2A). The 136 experiment was repeated twice. All the index hamsters had similar viral loads in the nasal 
137

138

139

140

141

142

143

144

145

146

147

148

149

150

151

152

153

154

155

156

157

158

159

turbinate at sacrifice at $2 \mathrm{dpi}$, indicating that they were successfully infected (Fig. 2B). All 12 naïve hamsters were found to be infected at sacrifice at 2 days after exposure (Fig. 2C), indicating that both variants are highly transmissible through close contact. Next, we randomly grouped 42 hamsters into 6 groups of index and naïve hamsters (1:6 ratio) in our established noncontact transmission system, and repeated the experiment twice (total $n=84)$ (Fig. 2D) (20). The hamsters were sacrificed at 2 dpi (index) or 2 days post-exposure to index (naïve). All the index hamsters were successfully infected with similarly high nasal turbinate virus titers (Fig. 2E). A total of $30 / 36(83.3 \%)$ and $24 / 36(66.7 \%)$ of naïve animals became infected by the Omicron and Delta variant, respectively, through non-contact transmission (Fig. 2F). Notably, although not yet reaching statistical significance with the sample size of 36 naïve hamsters per group $(\mathrm{P}=0.173)$, the transmission rate of Omicron variant was consistently about $10-20 \%$ higher than that of the Delta variant in both rounds of non-contact transmission experiments. This consistently higher transmission rate would likely be significant in real life scenario with large susceptible human populations.

\section{The Omicron variant outcompetes the Delta variant under immune selection pressure}

To investigate whether this highly transmissible Omicron variant is likely to take over as the dominant circulating SARS-CoV-2 variant, we performed a competition assay to compare its fitness with that of the presently circulating Delta variant. We co-infected human lung-derived Calu-3 cells with the Omicron and Delta variants. After 24 hours, we collected both culture supernatant and cell lysate, and quantified the relative amounts of RNA of the Omciron and Delta variants by quantitative reverse transcription-polymerase chain reaction (qRT-PCR) and Sanger sequencing. Both methods were validated to reliably quantify the relative amounts of the 
Omicron and Delta variants in mixed specimens (Fig. S7). Consistent with our recent preliminary findings at an early time-point, the Delta variant consistently exhibits significant fitness advantage over the Omicron variant for up to $72 \mathrm{hpi}$ in vitro (Fig. 3A and 3B) (21). However, this scenario drastically changed when selection pressure by vaccinated sera containing antibodies with reduced anti-Omicron but preserved anti-Delta neutralizing activity was present (Fig. 3C), with the Omicron variant significantly $(\mathrm{P}<0.0001)$ outcompeting the Delta variant (Fig. 3D). Then, we validated our in vitro findings with in vivo competition models in hamsters. We included both non-vaccinated (Fig. 3E) and vaccinated (Fig. 3F) index hamsters, and intranasally challenged them with the two variants (1:1 ratio). The vaccinated index hamsters were at 100 days post-vaccination with an inactivated SARS-CoV-2 vaccine and showed waning serum antibody response in comparison with the peak activity at 28 days post-vaccination (Fig.

172 than the Delta variant (Fig. 3H). In the non-vaccinated index hamsters, the Delta variant 173 significantly outcompeted the Omicron variant (Fig. 3I). In stark contrast, the Omicron variant 174 exhibited marked fitness advantage over the Delta variant in the vaccinated index hamsters (Fig. 175 3I). Regarding the naïve hamsters, the Delta variant similarly outcompeted the Omicron variant 176 in those that were exposed to non-vaccinated index hamsters (Fig. 3J). Whereas among the naïve 177 hamsters exposed to vaccinated index hamsters, the Omicron variant significantly outcompeted 178 the Delta variant (Fig. 3J). Overall, our findings demonstrated that the Delta variant exhibits 179 fitness advantage over the Omicron variant in the absence of selection pressure. Under immune 180 selection pressure, the Omicron variant significantly outcompetes the Delta variant to become 181 the dominant variant causing infection. 


\section{DISCUSSION}

184 Novel SARS-CoV-2 variants will continue to emerge as long as the virus maintains its wide circulation among humans and mammals. While it has recently become evident that the Omicron variant exhibits immune evasion to most existing anti-SARS-CoV-2 therapeutic monoclonal antibodies and vaccine-induced neutralizing antibodies (9-15), little is known about the in vivo pathogenicity, transmissibility, and fitness of this emerging VOC. The golden Syrian hamster model is a well-established animal model that closely mimics the clinical and virological features of COVID-19 in human, and has been widely applied for studying these aspects of SARS-CoV-2 $(19,20,22-26)$.

In our pathogenicity study, we showed that while the viral load and infectious virus titer of the two variants were similar in the nasal turbinate and trachea, the Omicron variant is

194 significantly less replicative in the lung even at the early post-infection stage (2 dpi). The 195 infected hamsters could more efficiently restrict replication of the Omicron variant than the Delta variant by 4 dpi and $7 \mathrm{dpi}$, with consistently less cytokine/chemokine dysregulation and tissue

197 damage from 2 dpi to 7 dpi. The lower anti-spike response in the Omicron-infected hamsters 198 corroborates with the overall less severe disease induced by the Omicron than the Delta variant.

199 Our in vivo findings corroborate with recent in vitro studies comparing Omicron with Delta and 200 other SARS-CoV-2 variants. In human lung-derived Calu-3 cells, the Omicron variant shows 201 lower replication than the Delta variant and D614G strain in in pseudovirus and/or live virus 202 assays $(21,27)$. Moreover, the Omicron spike exhibits reduced receptor binding, fusogenicity, as 203 well as S1 subunit shedding in vitro $(21,27)$. Interestingly, while our data indicated that the 204 Omicron variant is generally less replicative than the Delta variant throughout the upper and 205 lower respiratory tract, a recent preprint non-peer-reviewed study using ex vivo lung organ 
culture reported the Omicron variant showed higher replication than the Delta variant in the bronchi (28). This apparent difference may be caused by the different study models and conditions. It is also notable that there are significant variations in the ex vivo bronchi results which may have been obtained from different patient donors. Taken together, our in vivo findings help to explain the observations in early epidemiological studies reporting lower rates of hospitalization caused by the Omicron variant than the Delta variant. Preliminary analysis in South Africa reported that the hospitalization rate was about 30\% lower among Omicroninfected patients than those infected with previous variant (16). Preliminary estimation in the United Kingdom also showed that the hospitalization rate of Omicron-infected patients may be $40-45 \%$ lower than that of Delta-infected patients (29). Importantly, we also showed that virus shedding in the oral swabs and feces were both significantly lower and shorter in the Omicroninfected than Delta-infected hamsters. This may have implications on the management and infection control of Omicron-infected patients should the same viral shedding pattern be confirmed in human.

The transmissibility of the Omicron variant is another key factor for optimizing public health control measures and predicting the evolvement of the pandemic. Recent epidemiological studies suggested that the Omicron variant may be spreading even faster (up to 4.2 times) than the Delta variant in its early stage (30). The estimated $\mathrm{R}_{\mathrm{t}}$ of the Omicron variant in South Africa and the United Kingdom is 2.5 to 3.7 , with a doubling time of approximately every 3 days (31). Our head-to-head comparison in this hamster model showed that that the Omicron variant has at least non-inferior, if not higher, transmissibility than the Delta variant in both contact (100\% vs $100 \%$ after 4 hours of close contact) and non-contact ( $83.3 \%$ vs $66.7 \%$ after 6 hours of exposure) of transmission. The lack of statistical significance in our study was likely related to the use of 
the Delta variant which is highly transmissible itself as the comparator. It is noteworthy that the $15-20 \%$ higher rate of transmission through non-contact transmission would likely be epidemiologically significant if the same degree of enhanced transmission was found in large

232 human populations, especially in areas with low herd immunity and relaxed public health control measures. This non-inferior or even higher transmissibility of the Omicron variant despite generally lower respiratory tract viral load than the Delta variant in our hamster model and in Calu-3 cells in vitro (21) suggests that other factors such as the efficiency of the variants to enter cells, and their ability to maintain as infectious particles in aerosols for prolonged periods should be further investigated. The functional role of the amino acid mutations found in the Omicron but not the Delta variant, for example the N501Y mutation that may be associated with enhanced transmissibility, should be further characterized (32).

to become the dominant SARS-CoV-2 strain, we compared their in vitro and in vivo fitness in cell culture and hamster models. Rather unexpectedly, the rapidly disseminating Omicron variant was consistently outcompeted by the Delta variant in vitro and in unvaccinated hamsters, which 244 might be the results of its unusually high number of genetic mutations. More importantly, we 245 showed that the Omicron variant exhibits significant fitness advantage over the Delta variant 246 under selection pressure in vitro in the presence of vaccinated serum and in hamsters with 247 waning serum neutralizing antibody level at 100 days post-vaccination with inactivated COVID24819 vaccine. Taken together, our findings suggest that the Omicron variant may outcompete the 249 Delta variant to become the predominant SARS-CoV-2 strain especially in populations with high rates of previous infection and/or vaccination with first-generation COVID-19 vaccines eliciting 251 ineffective neutralizing antibody response against the Omicron variant. However, our findings 
252 should be carefully interpreted and not be considered as evidence against COVID-19

253 vaccination. On the contrary, our findings in hamsters with waning serum neutralizing antibody

254 at more than 3 months after vaccination are supportive of booster vaccines because recent

255 preprint data have shown that antibody neutralization is mostly restored by mRNA vaccine

256 booster doses (33).

257 Our study had limitations. The transmission rate of SARS-CoV-2 may vary according to

258 different durations of exposure. In this study, we selected 6 hours of non-contact transmission to

259 simulate the scenarios of staying with an infected index patient within the same facility for a

260 routine business day and on medium-haul flights. It would be worthwhile to further compare the

261 transmissibility of the Omicron and other variants after different durations of exposure in future

262 studies. It would also be important to investigate the pathogenicity and transmissibility of the

263 Omicron variant in additional animal models such as the hACE2-transgenic mouse and non-

264 human primate models, as each of these animal models have their advantages and disadvantages

265 in recapitulating human disease.

In summary, the present study shows that despite comparatively lower pathogenicity than

267 the Delta variant, the Omicron variant undoubtedly still causes obvious clinical effects, increased

268 viral burdens and pro-inflammatory cytokines/chemokines, as well as histopathological damages

269 in infected hosts. Taking into consideration the Omicron variant's higher transmissibility than

270 the already-highly transmissible Delta variant, our findings highlight the urgent need to find

271 next-generation COVID-19 vaccines and broad-spectrum therapeutics, as well as to tighten non-

272 pharmaceutical measures to reduce acute and chronic disease burden (long COVID) on the

273 public and healthcare facilities. 


\section{MATERIALS AND METHODS}

\section{Study design}

277 The emerging Omicron variant has an alarmingly high number of mutations in its spike, which

278 may not only affect its susceptibility to existing vaccines and monoclonal antibodies, but also its

279 pathogenicity, transmission, and fitness. This study was designed to characterize these important

280 virological attributes of the Omicron variant. To optimally place our findings into the context of

281 the latest COVID-19 pandemic development, we performed side-by-side comparison between

282 the Omicron variant and the currently dominant Delta variant. For the in vivo experiments, we

283 adopted the well-established golden Syrian hamster COVID-19 model which closely mimics

284 human disease (19). Gender- and age-matched hamsters were randomized into different

285 experimental groups. The group sizes were chosen based on statistical power analysis and our

286 prior experience in examining virus burdens and cytokine/chemokine profiles in hamsters. The

287 hamsters were challenged with either SARS-CoV-2 variant and the blood and tissues were

288 harvested for virological, cytokine/chemokine, and histopathological studies. Body weights and

289 clinical scores were monitored until the indicated time-points. For the measurement of

290 quantitative values, such as viral genome copies, infectious viral titers, cytokine/chemokine gene

291 copies, body weights and clinical scores, no blinding procedures were applied to the

292 experimentalists involved. For the histological examinations, qualified pathologists were blinded

293 to group allocation to ensure the assessment was unbiased. All data collected was included

294 without exclusion of outliers. All experiments in mice were individually performed in two

295 separate occasions. For the in vitro competition experiment, live virus infection assays were

296 performed to delineate the replication of the Omicron and Delta variants in human lung-derived 
297

298

299

300

301

302

303

304

305

306

307

308

309

310

311

312

313

314

315

316

317

Calu3 cells. Viral loads and infectious virus titers were quantified with RT-qPCR and plaque assays, respectively.

Viruses and cells. SARS-CoV-2 Omicron (hCoV-19/Hong Kong/HKU-344/2021; GISAID accession number EPI_ISL_7357684) and Delta (hCoV-19/Hong Kong/HKU-210804-001/2021; GISAID: EPI_ISL_3221329) variants were isolated from respiratory tract specimens of laboratory-confirmed COVID-19 patients in Hong Kong (9). The inoculated cells were monitored daily for cytopathic effects by light microscopy and the cell supernatants were collected daily for qRT-PCR to assess viral load. The viruses were passaged two times before being used for the experiments. The cell lines used in this study were available in our laboratory as previously described (34). Calu-3 and VeroE6-TMPRSS2 cells were maintained in DMEM culture medium supplemented with $10 \%$ heat-inactivated FBS, $50 \mathrm{U} / \mathrm{ml}$ penicillin and $50 \mu \mathrm{g} / \mathrm{ml}$ streptomycin. All experiments involving live SARS-CoV-2 followed the approved standard operating procedures of the Biosafety Level 3 facility at The University of Hong Kong $(19,20,35)$.

In vivo pathogenicity study in golden Syrian hamsters. The pathogenicity of the Omicron and Delta variants were compared in the established golden Syrian hamster model for COVID-19 as we described previously (19). Briefly, male and female hamsters, aged 8-10 weeks old, were obtained from the Chinese University of Hong Kong Laboratory Animal Service Centre through the HKU Centre for Comparative Medicine Research. The hamsters were kept in biosafety level 2 housing and given access to standard pellet feed and water ad libitum. At 0 dpi, each hamster was intranasally inoculated with $100 \mu \mathrm{L}$ of DMEM containing $10^{5}$ PFU of SARS-CoV-2 $(n=15$ 
320 for each variant) under intraperitoneal ketamine $(200 \mathrm{mg} / \mathrm{kg})$ and xylazine $(10 \mathrm{mg} / \mathrm{kg})$ anesthesia.

321 The body weight and clinical signs of disease of the hamsters were monitored daily. A score of 1

322 was given to each of the following clinical signs: lethargy, ruffled fur, hunchback posture, and

323 rapid breathing as previously described (36). The hamsters were sacrificed at $2 \mathrm{dpi}, 4 \mathrm{dpi}$, and 7

324 dpi (n=5 per variant per time-point) for viral load quantitation by qRT-PCR, virus titer

325 quantitation by plaque assay, histopathological studies and immunofluorescent staining as

326 described previously (19,37). Additional hamsters ( $\mathrm{n}=5$ per variant) were kept beyond 7 dpi for

327 serial viral load detection in oral swabs and feces.

SARS-CoV-2 spike RBD binding assay. Microwell plates were coated with $100 \mathrm{ng} / \mathrm{well}$ of

SARS-CoV-2 RBD specific for each variant (Omicron RBD, SinoBiological \#Cat:40592-

by incubation with blocking reagent overnight at $4{ }^{\circ} \mathrm{C}$. After removal of the blocking solution, washed 6 times, rabbit anti-hamster horseradish peroxidase antibody $(100 \mu \mathrm{L} /$ well $)$ at the

337 times, tetramethylbenzidine substrate $(100 \mu \mathrm{L} /$ well $)$ was added. The reaction was stopped after

33810 min by the addition of $0.3 \mathrm{~N}$ sulfuric acid, and the plates were then examined in an ELISA 339 plate at $450 \mathrm{~nm}$ and $620 \mathrm{~nm}$. previously (19). Briefly, Index hamsters were intranasally challenged with either the Omicron or 
343 Delta variant at 0 dpi. Twenty-four hours later, each virus-challenged index hamster was

344 transferred to a new cage, with each cage containing one naïve hamster as a close contact. The

345 index and naïve hamsters were co-housed for 4 hours before transferral to new cages. The index

346 and contact hamsters were sacrificed at 2 dpi and 2 days post-exposure, respectively. Hamsters

347 with cycle threshold $(\mathrm{Ct})$ value $<40$ in either nasal turbinate or lung were considered as infected.

348 The experiment was repeated twice, each time with 6 index $(n=3$ for Omicron and $n=3$ for Delta

349 each time) and 6 naïve hamsters ( $n=3$ for Omicron and $n=3$ for Delta each time).

351 Non-contact transmission study. The transmissibility of the Omicron and Delta variants among

352 hamsters through non-contact transmission was compared in our established closed system with

353 unidirectional airflow in two independent experiments (20). In each experiment, 6 index

354 hamsters (n=3 for each variant), each housed in a separate cage (Marukan Co., Ltd., Osaka,

355 Japan) inside isolators (Tecniplast SpA, Varese, Italy), were intranasally challenged with $100 \mu \mathrm{L}$

356 of Dulbecco's Modified Eagle Medium (DMEM) containing $10^{5}$ plaque-forming units of either

357 variant under intraperitoneal ketamine and xylazine anaesthesia at 0 dpi. After $24 \mathrm{~h}, 36$ naïve

358 hamsters were transferred to cages adjacent to the cages housing the index hamsters (1:6 ratio).

359 The index and exposed naïve hamsters were removed from the non-contact transmission system

360 and transferred to separate new cages 6 hours later. The hamsters were then sacrificed at 2 dpi

361 (index) or 2 days post-exposure (naïve) for organ tissue collection. Hamsters with Ct value $<40$

362 in either nasal turbinate or lung were considered as infected. The experiment was repeated twice, 363 with a total of 12 index and 72 naïve hamsters. 
365 In vitro virus competition assay. Approximately $3 \times 10^{5}$ cells were seeded onto each well of $12-$

366 well plates and cultured at $37^{\circ} \mathrm{C}, 5 \% \mathrm{CO}_{2}$ for $16 \mathrm{~h}$. Equal PFUs of two variants (1:1 ratio) were

367 inoculated onto Calu-3 cells at a final MOI of 0.10 for each variant. The mixed viruses were

368 incubated with the cells at $37^{\circ} \mathrm{C}$ for $2 \mathrm{~h}$. After infection, the cells were washed twice with PBS to

369 remove residual viruses. One milliliter of culture medium was added into each well. At each

370 time-point, 350 $\mu 1$ cell culture lysate and/or supernatant was collected for RNA extraction. Ratios

371 of Omicron:Delta RNA were determined via RT-PCR with quantification of Sanger peak heights

372 or via qRT-PCR using specific primer and probes. All samples were stored at $-80^{\circ} \mathrm{C}$ until

373 analysis.

374

375 In vivo virus competition models. To evaluate the competitive fitness of the Omicron and Delta

376 variants in vivo, virus competition experiments were performed using non-vaccinated and

377 vaccinated hamsters. In the non-vaccinated hamster model, six index hamsters were infected

378 each intranasally with $10^{5}$ PFU of a mixture of both viruses (1:1 ratio). At 2 dpi, each index

379 hamster was co-housed with one naïve hamsters for 4 hours in six independent lines of naïve

380 animals. Total RNA was extracted from the nasal turbinate, trachea, and lung tissues, of the

381 hamsters at 2 dpi or 2 days post-exposure (exposed naïve) using RNeasy kit (Qiagen) for viral

382 load quantification and calculation of the Omicron:Delta ratio. In the vaccinated hamster model,

383 each index hamster was intramuscularly administered with $3 \mu \mathrm{g}$ per dose of inactivated vaccine at

384 day 0 and boosted at day 14, respectively. To generate the inactivated virus vaccine, virus-

385 containing supernatant [SARS-CoV-2 virus HKU-001a strain, GenBank accession number:

386 MT230904)] was harvested and inactivated using $0.2 \%$ formaldehyde solution in PBS for 5 days.

387 Cell cytopathic effects (CPE) were monitored to validate complete inactivation using Vero cells. 
Hamster sera were collected at day 0 , day 14 , day 28, and day 100 for neutralization antibody titration using enzyme-linked immunosorbent assay and micro-neutralization assay. At day 100 post-vaccination, the vaccinated index hamsters were infected (ie: 0dpi) and the competition experiments were performed as described for the non-vaccinated hamster model.

Validation of competition assay. The experiments were performed as previously described (26). To validate the consistency and accuracy of the competition assay in both system, the Omicron and Delta variants were mixed at ratios of 10:1, 5:1, 3:1, 1:1, 1:3, 1:5, and 1:10 based on their PFU titres (total $10^{5}$ PFU viruses), or mixed with $10^{6}, 10^{5}, 10^{4}, 10^{3}$, and $10^{2} \mathrm{PFU}$ of the two variants at a ratio of $1: 1$. The total RNA of these mixed variants was isolated and amplified by RT-PCR followed by Sanger sequencing, or directly analysed by one-step RT-qPCR using strain-specific primers and probes as mentioned below. The Omicron to Delta ratio was

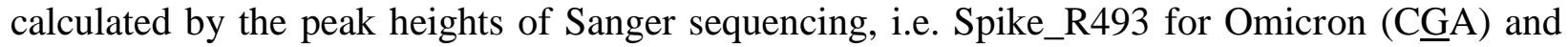
Spike_Q493 for Delta (CAAA), or the copy numbers by the RT-qPCR system targeting a spike region of Omicron and Delta. Data were analysed by linear regression with correlation coefficients (r) and significance $(\mathrm{P})$.

Quantification of RNA ratios between Omicron and Delta variants. To measure the ratios Omicron to delta for competition assays, both qRT-PCR and Sanger sequencing were performed. Real-time one-step qRT-PCR was used for quantitation of Omicron and Delta spike gene copy within the same input template after RNA extraction. The QuantiNova Probe RT-PCR kit (Qiagen) was used with a LightCycler 480 Real-Time PCR System (Roche) as previously described (38). Each $20 \mu \mathrm{L}$ reaction mixture contained $10 \mu \mathrm{L}$ of $2 \times$ QuantiNova Probe RT-PCR 
411 Master Mix, $0.8 \mu \mathrm{L}$ of RNase-free water, $0.2 \mu \mathrm{L}$ of QuantiNova Probe RT-Mix, $0.8 \mu \mathrm{L}$ each of

$41220 \mu \mathrm{M}$ forward and reverse primers, $0.4 \mu \mathrm{L}$ each of $10 \mu \mathrm{M}$ probes, and $5 \mu \mathrm{L}$ of extracted RNA as

413 the template. Reactions were incubated at $45^{\circ} \mathrm{C}$ for $10 \mathrm{~min}$ for reverse transcription, $95^{\circ} \mathrm{C}$ for 5

$414 \min$ for denaturation, followed by 45 cycles of $95^{\circ} \mathrm{C}$ for $5 \mathrm{~s}$ and $55^{\circ} \mathrm{C}$ for $30 \mathrm{~s}$. Signal detection

415 and measurement were taken in each cycle after the annealing step. The cycling profile ended

416 with a cooling step at $40^{\circ} \mathrm{C}$ for 30 s. Primers and probes to be used are as below: Omi-S_F:

417 ACAAACCTTGTAATGGTGTTGC; Omi-S_R: TACTACTACTCTGTATGGTTGGTG; Omi-

418 S_probe: Cy5-CGATCATATAGTTTCCGACCCACTT-IAbRQSp; Delta-S_F:

419 GCAAACCTTGTAATGGTGTTGA; Delta-S_R: GTACTACTACTCTGTATGGTTGGTA;

420 Delta-S_probe: FAM- CAATCATATGGTTTCCAACCCACTA -IABkFQ. For Sanger

421 sequencing, RT-PCR was conducted using a SuperScript ${ }^{\mathrm{TM}}$ III One-Step RT-PCR kit

422 (Invitrogen, Carlsbad, CA, USA) to amplify the extracted viral RNA (Qiagen). Relative

423 replicative fitness values for omicron virus compared to delta virus were analysed according to

$424 w=(f O / i 0)$, where $i 0$ was the initial Omicron/Delta ratio and $f O$ was the final Omicron/Delta ratio

425 after competition. Sanger sequencing (initial time-point T0) counts for each variant being

426 compared were based upon average counts over repeated samples of inoculum per experiment,

427 and post-infection (time-point T1) counts were taken from samples of individual subjects. To

428 model fO/iO, the ratio T0/T1 was determined for each subject in distinct strain groups.

430 Statistical analysis. All data were analysed with GraphPad Prism software (GraphPad Software,

431 Inc). Body weight losses were compared using two-way ANOVA. Two-way ANOVA or

432 Student's t-test was used to determine significant differences in viral loads and titers. $\mathrm{P}<0.05$ was

433 considered statistically significant. 
435 Ethical approvals. The animal experiments were approved by the Institutional Review Board of

436 The University of Hong Kong Committee on the Use of Live Animals in Teaching and Research

437 (CULATR) and the use of clinical specimens was approved by the Institutional Review Board of 438 the University of Hong Kong / Hospital Authority Hong Kong West Cluster.

440 Illustrations. The hamster illustrations and schematic figures were created with BioRender 441 software (https://biorender.com/).

443 Supplementary Materials.

444 Fig. S1. Virus shedding in the oral swabs and feces of the Omicron-infected and Delta-infected 445 hamsters.

446 Fig. S2. Variant-specific anti-spike receptor-binding domain antibody response of the Omicron447 infected and Delta-infected hamsters.

448 Fig. S3. Representative histopathological findings of the lung of Omicron-infected and Delta449 infected hamsters.

450 Fig. S4. Representative immunofluorescence staining findings of the lung of Omicron-infected 451 and Delta-infected hamsters.

452 Fig. S5. Representative histopathological findings of the nasal turbinate of Omicron-infected and 453 Delta-infected hamsters.

454 Fig. S6. Representative immunofluorescence staining findings of the nasal turbinate of Omicron455 infected and Delta-infected hamsters.

456 Fig. S7. Validation of the competition assay. 


\section{REFERENCES}

458 1. Cohen J, Normile D. New SARS-like virus in China triggers alarm. Science. 367, 234-235

459 (2020).

460 2. Zhou P, Yang XL, Wang XG, Hu B, Zhang L, Zhang W, Si HR, Zhu Y, Li B, Huang CL,

461 Chen HD, Chen J, Luo Y, Guo H, Jiang RD, Liu MQ, Chen Y, Shen XR, Wang X, Zheng XS,

462 Zhao K, Chen QJ, Deng F, Liu LL, Yan B, Zhan FX, Wang YY, Xiao GF, Shi ZL, A pneumonia

463 outbreak associated with a new coronavirus of probable bat origin. Nature. 579, 270-273 (2020).

464 3. Chan JF, Yuan S, Kok KH, To KK, Chu H, Yang J, Xing F, Liu J, Yip CC, Poon RW, Tsoi 465 HW, Lo SK, Chan KH, Poon VK, Chan WM, Ip JD, Cai JP, Cheng VC, Chen H, Hui CK, Yuen

466 KY. A familial cluster of pneumonia associated with the 2019 novel coronavirus indicating

467 person-to-person transmission: a study of a family cluster. Lancet. 395, 514-523 (2020).

468 4. To KK, Sridhar S, Chiu KH, Hung DL, Li X, Hung IF, Tam AR, Chung TW, Chan JF, Zhang

469 AJ, Cheng VC, Yuen KY. Lessons learned 1 year after SARS-CoV-2 emergence leading to

470 COVID-19 pandemic. Emerg Microbes Infect. 10, 507-535 (2021).

471 5. Davies NG, Abbott S, Barnard RC, Jarvis CI, Kucharski AJ, Munday JD, Pearson CAB,

472 Russell TW, Tully DC, Washburne AD, Wenseleers T, Gimma A, Waites W, Wong KLM, van

473 Zandvoort K, Silverman JD; CMMID COVID-19 Working Group; COVID-19 Genomics UK

474 (COG-UK) Consortium, Diaz-Ordaz K, Keogh R, Eggo RM, Funk S, Jit M, Atkins KE,

475 Edmunds WJ. Estimated transmissibility and impact of SARS-CoV-2 lineage B.1.1.7 in England.

$476 \quad$ Science. 372, eabg3055 (2021).

477 6. Ulrich L, Halwe NJ, Taddeo A, Ebert N, Schön J, Devisme C, Trüeb BS, Hoffmann B, Wider

478 M, Fan X, Bekliz M, Essaidi-Laziosi M, Schmidt ML, Niemeyer D, Corman VM, Kraft A,

479 Godel A, Laloli L, Kelly JN, Calderon BM, Breithaupt A, Wylezich C, Veiga IB, Gultom M, 
480

481

482

483

484

485

486

487

488

489

490

491

492

493

494

495

496

497

498

499

500

501

Osman S, Zhou B, Adea K, Meyer B, Eberhardt C, Thomann L, Gsell M, Labroussaa F, Jores J, Summerfield A, Drosten C, Eckerle IA, Wentworth DE, Dijkman R, Hoffmann D, Thiel V, Beer M, Benarafa C. Enhanced fitness of SARS-CoV-2 variant of concern Alpha but not Beta. Nature. 2021 Dec 22. doi: 10.1038/s41586-021-04342-0 (2021).

7. World Health Organization. Classification of Omicron (B.1.1.529): SARS-CoV-2 Variant of Concern. https://www.who.int/news/item/26-11-2021-classification-of-omicron-(b.1.1.529)-sars-

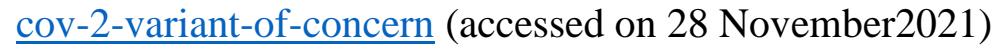

8. World Health Organization. Update on Omicron. https://www.who.int/news/item/28-11-2021update-on-omicron (accessed on 30 November 2021)

9. Lu L, Mok BW, Chen LL, Chan JM, Tsang OT, Lam BH, Chuang VW, Chu AW, Chan WM, Ip JD, Chan BP, Zhang R, Yip CC, Cheng VC, Chan KH, Jin DY, Hung IF, Yuen KY, Chen H, To KK. Neutralization of SARS-CoV-2 Omicron variant by sera from BNT162b2 or Coronavac vaccine recipients. Clin Infect Dis. 2021 Dec 16:ciab1041. doi: 10.1093/cid/ciab1041 (2021).

10. Liu L, Iketani S, Guo Y, Chan JF, Wang M, Liu L, Luo Y, Chu H, Huang Y, Nair MS, Yu J, Chik KK, Yuen TT, Yoon C, To KK, Chen H, Yin MT, Sobieszczyk ME, Huang Y, Wang HH, Sheng Z, Yuen KY, Ho DD. Striking antibody evasion manifested by the Omicron variant of SARS-CoV-2. Nature. 2021 Dec 23. doi: 10.1038/d41586-021-03826-3 (2021).

11. Cele S, Jackson L, Khoury DS, Khan K, Moyo-Gwete T, Tegally H, San JE, Cromer D, Scheepers C, Amoako D, Karim F, Bernstein M, Lustig G, Archary D, Smith M, Ganga Y, Jule Z, Reedoy K, Hwa SH, Giandhari J, Blackburn JM, Gosnell BI, Abdool Karim SS, Hanekom W, Network for Genomic Surveillance in South Africa, COMMIT-KZN Team, von Gottberg A, Bhiman J, Lessells RJ, Moosa MS, Davenport MP, de Oliveira T, Moore PL, Sigal A. Omicron 
extensively but incompletely escapes Pfizer BNT162b2 neutralization. Nature. 2021 Dec 23. doi: 10.1038/d41586-021-03824-5 (2021).

12. Cao Y, Wang J, Jian F, Xiao T, Song W, Yisimayi A, Huang W, Li Q, Wang P, An R, Wang J, Wang Y, Niu X, Yang S, Liang H, Sun H, Li T, Yu T, Cui Q, Liu S, Yang X, Du X, Zhang Z, Hao X, Shao F, Jin R, Wang X, Xiao J, Wang Y, Xie XS. Omicron escapes the majority of existing SARS-CoV-2 neutralizing antibodies. Nature. 2021 Dec 23. doi: 10.1038/d41586-02103796-6 (2021).

13. Planas D, Saunders N, Maes P, Guivel-Benhassine F, Planchais C, Buchrieser J, Bolland W, Porrot F, Staropoli I, Lemoine F, Péré H, Veyer D, Puech J, Rodary J, Baela G, Dellicour S, Raymenants J, Gorissen S, Geenen C, Vanmechelen B, Wawina-Bokalanga T, Martí-Carrerasi J, Cuypers L, Sève A, Hocqueloux L, Prazuck T, Rey F, Simon-Lorrière E, Bruel T, Mouquet H, André E, Schwartz O. Considerable escape of SARS-CoV-2 Omicron to antibody neutralization. Nature. 2021 Dec 23. doi: 10.1038/d41586-021-03827-2 (2021).

14. Cameroni E, Bowen JE, Rosen LE, Saliba C, Zepeda SK, Culap K, Pinto D, VanBlargan LA, De Marco A, di Iulio J, Zatta F, Kaiser H, Noack J, Farhat N, Czudnochowski N, HavenarDaughton C, Sprouse KR, Dillen JR, Powell AE, Chen A, Maher C, Yin L, Sun D, Soriaga L, Bassi J, Silacci-Fregni C, Gustafsson C, Franko NM, Logue J, Iqbal NT, Mazzitelli I, Geffner J, Grifantini R, Chu H, Gori A, Riva A, Giannini O, Ceschi A, Ferrari P, Cippà PE, FranzettiPellanda A, Garzoni C, Halfmann PJ, Kawaoka Y, Hebner C, Purcell LA, Piccoli L, Pizzuto MS, Walls AC, Diamond MS, Telenti A, Virgin HW, Lanzavecchia A, Snell G, Veesler D, Corti D. Broadly neutralizing antibodies overcome SARS-CoV-2 Omicron antigenic shift. Nature. 2021 Dec 23. doi: 10.1038/d41586-021-03825-4 (2021). 
524 15. Ai J, Zhang H, Zhang Y, Lin K, Zhang Y, Wu J, Wan Y, Huang Y, Song J, Fu Z, Wang H,

525 Guo J, Jiang N, Fan M, Zhou Y, Zhao Y, Zhang Q, Liu Q, Lv J, Li P, Qiu C, Zhang W. Omicron

526 variant showed lower neutralizing sensitivity than other SARS-CoV-2 variants to immune sera

527 elicited by vaccines after boost. Emerg Microbes Infect. 2021 Dec 22:1-24. doi:

$528 \quad 10.1080 / 22221751.2021 .2022440(2021)$.

529 16. Ledford H. How severe are Omicron infections? Nature.

530 https://www.nature.com/articles/d41586-021-03794-8 (accessed on 18 December 2021).

531 17. Espenhain L, Funk T, Overvad M, Edslev SM, Fonager J, Ingham AC, Rasmussen M,

532 Madsen SL, Espersen CH, Sieber RN, Stegger M, Gunalan V, Wilkowski B, Larsen NB, Legarth

533 R, Cohen AS, Nielsen F, Lam JUH, Lavik KE, Karakis M, Spiess K, Marving E, Nielsen C,

534 Wiid Svarrer C, Bybjerg-Grauholm J, Olsen SS, Jensen A, Krause TG, Müller L.

535 Epidemiological characterisation of the first 785 SARS-CoV-2 Omicron variant cases in

536 Denmark, December 2021. Euro Surveill. 2021 Dec;26(50). doi: 10.2807/1560-

537 7917.ES.2021.26.50.2101146 (2021).

538 18. Brandal LT, MacDonald E, Veneti L, Ravlo T, Lange H, Naseer U, Feruglio S, Bragstad K,

539 Hungnes O, Ødeskaug LE, Hagen F, Hanch-Hansen KE, Lind A, Watle SV, Taxt AM, Johansen

540 M, Vold L, Aavitsland P, Nygård K, Madslien EH. Outbreak caused by the SARS-CoV-2

541 Omicron variant in Norway, November to December 2021. Euro Surveill. 2021 Dec;26(50). doi:

$542 \quad$ 10.2807/1560-7917.ES.2021.26.50.2101147 (2021).

543 19. Chan JF, Zhang AJ, Yuan S, Poon VK, Chan CC, Lee AC, Chan WM, Fan Z, Tsoi HW, Wen

544 L, Liang R, Cao J, Chen Y, Tang K, Luo C, Cai JP, Kok KH, Chu H, Chan KH, Sridhar S, Chen

545 Z, Chen H, To KK, Yuen KY. Simulation of the Clinical and Pathological Manifestations of 
Coronavirus Disease 2019 (COVID-19) in a Golden Syrian Hamster Model: Implications for

Disease Pathogenesis and Transmissibility. Clin Infect Dis. 71, 2428-2446 (2020).

K, Luo C, Cheng VC, Cai JP, Chu H, Chan KH, To KK, Sridhar S, Yuen KY. Surgical Mask

Jin DY, Chen H, Yuen KY, To KK. SARS-CoV-2 Omicron variant shows less efficient 
25. de Melo GD, Lazarini F, Levallois S, Hautefort C, Michel V, Larrous F, Verillaud B,

570 Aparicio C, Wagner S, Gheusi G, Kergoat L, Kornobis E, Donati F, Cokelaer T, Hervochon R,

571 Madec Y, Roze E, Salmon D, Bourhy H, Lecuit M, Lledo PM. COVID-19-related anosmia is

572 associated with viral persistence and inflammation in human olfactory epithelium and brain

573 infection in hamsters. Sci Transl Med. 13, eabf8396 (2021).

574 26. Plante JA, Liu Y, Liu J, Xia H, Johnson BA, Lokugamage KG, Zhang X, Muruato AE, Zou

575 J, Fontes-Garfias CR, Mirchandani D, Scharton D, Bilello JP, Ku Z, An Z, Kalveram B, Freiberg

576 AN, Menachery VD, Xie X, Plante KS, Weaver SC, Shi PY. Spike mutation D614G alters

577 SARS-CoV-2 fitness. Nature. 592, 116-121 (2021).

578 27. Zeng C, Evans JP, Qu P, Faraone J, Zheng YM, Carlin C, Bednash JS, Zhou T, Lozanski G,

579 Mallampalli R, Saif LJ, Oltz EM, Mohler P, Xu K, Gumina RJ, Liu SL. Neutralization and 580 stability of SARS-CoV-2 Omicron variant. bioRxiv. doi.org/10.1101/2021.12.16.472934 (2021).

581 28. HKUMed News. HKUMed finds Omicron SARS-CoV-2 can infect faster and better than 582 Delta in human bronchus but with less severe infection in lung.

583 https://www.med.hku.hk/en/news/press/20211215-omicron-sars-cov-2-infection

584 (accessed on 16 December 2021).

585 29. Imperial College London. Report 50 - Hospitalisation risk for Omicron cases in England 586 https://www.imperial.ac.uk/mrc-global-infectious-disease-analysis/covid-19/report-50-severity-

587 omicron/ (2021) (accessed on 25 December 2021).

588 30. Nishiura H, Ito K, Anzai A, Kobayashi T, Piantham C, Rodriguez-Morales AJ. Relative 589 reproduction number of SARS-CoV-2 Omicron (B.1.1.529) compared with Delta variant in 590 South Africa. J Clin Med. 11, 30 (2022). 

and priority actions for member states. 17 December 2021. https://www.who.int/docs/defaultsource/coronaviruse/20211217-global-technical-brief-and-priority-action-on-omicron_latest-

594 2.pdf?sfvrsn=bdd8297c_9\&download=true (accessed on 19 December 2021). 04245-0 (2021).

33. Gardner BJ, Kilpatrick AM. Estimates of reduced vaccine effectiveness against hospitalization, infection, transmission and symptomatic disease of a new SARS-CoV-2 variant,

medRxiv. doi:

603 34. Chu H, Chan JF, Yuen TT, Shuai H, Yuan S, Wang Y, Hu B, Yip CC, Tsang JO, Huang X, replication kinetics, and cell damage profiling of SARS-CoV-2 and SARS-CoV with

607 implications for clinical manifestations, transmissibility, and laboratory studies of COVID-19: an 608 observational study. Lancet Microbe. 1, e14-e23 (2020).

610 Poon VK, Lee AC, Matsunaga N, Pu Y, Yuen CK, Cao J, Liang R, Tang K, Sheng L, Du Y, Xu 611 W, Lau CY, Sit KY, Au WK, Wang R, Zhang YY, Tang YD, Clausen TM, Pihl J, Oh J, Sze KH, 
613 DY, Sun R, Chanda SK, Yuen KY. Clofazimine broadly inhibits coronaviruses including SARS-

614 CoV-2. Nature. 593, 418-423 (2021).

615 36. Lee AC, Zhang AJ, Chan JF, Li C, Fan Z, Liu F, Chen Y, Liang R, Sridhar S, Cai JP, Poon

616 VK, Chan CC, To KK, Yuan S, Zhou J, Chu H, Yuen KY. Oral SARS-CoV-2 Inoculation

617 Establishes Subclinical Respiratory Infection with Virus Shedding in Golden Syrian Hamsters.

618 Cell Rep Med. 1, 100121 (2020).

619 37. Yuan S, Wang R, Chan JF, Zhang AJ, Cheng T, Chik KK, Ye ZW, Wang S, Lee AC, Jin L,

620 Li H, Jin DY, Yuen KY, Sun H. Metallodrug ranitidine bismuth citrate suppresses SARS-CoV-2

621 replication and relieves virus-associated pneumonia in Syrian hamsters. Nat Microbiol. 5, 1439-

$6221448(2020)$.

623 38. Chan JF, Yip CC, To KK, Tang TH, Wong SC, Leung KH, Fung AY, Ng AC, Zou Z, Tsoi

624 HW, Choi GK, Tam AR, Cheng VC, Chan KH, Tsang OT, Yuen KY. Improved Molecular

625 Diagnosis of COVID-19 by the Novel, Highly Sensitive and Specific COVID-19-RdRp/Hel

626 Real-Time Reverse Transcription-PCR Assay Validated In Vitro and with Clinical Specimens. $J$

627 Clin Microbiol. 58, e00310-20 (2020). 


\section{ACKNOWLEDGEMENTS}

629 Funding: This study was partly supported by funding to The University of Hong Kong: the Health and Medical Research Fund (COVID1903010 - Project 6 and Project 7), the Food and

(D.-Y.J.); the Consultancy Service for Enhancing Laboratory Surveillance of Emerging

Infectious Diseases and Research Capability on Antimicrobial Resistance for Department of

National Program on Key Research Project of China (2020YFA0707500 and 2020YFA0707504)

J.F.-W.C.); the High Level-Hospital Program, Health Commission of Guangdong Province,

(J.F.-W.C.); National Key Research and Development Programme on Public Security Risk

647 Guangzhou Laboratory (EKPG22-01) (K.-Y.Y., J.-F.W.C.); and donations from the Shaw 648 Foundation Hong Kong, the Richard Yu and Carol Yu, Michael Seak-Kan Tong, May Tam Mak 
651 Chow Sin Lan Charity Fund Limited, Chan Yin Chuen Memorial Charitable Foundation, Marina

652 Man-Wai Lee, the Hong Kong Hainan Commercial Association South China Microbiology

653 Research Fund, the Jessie \& George Ho Charitable Foundation, Perfect Shape Medical Limited,

654 Kai Chong Tong, Tse Kam Ming Laurence, Foo Oi Foundation Limited, Betty Hing-Chu Lee,

655 Ping Cham So, and Lo Ying Shek Chi Wai Foundation. The funding sources had no role in the 656 study design, data collection, analysis, interpretation, or writing of the report.

657

658 Author contributions:

659 Conceptualization: S.Y., K.-Y.Y., J.F.-W.C.

660 Methodology: S.Y., D.-Y.J., J.F.-W.C.

661 Investigation: S.Y., Z.W.Y., R.L., K.T., A.Z., G.L., C.P.O., V.K.-M.P., C.C.-S.C., B.W.-Y.M., 662 Z.Q., Y.X., H.S., J.O.-L.T., T.T.-T.Y., K.K.-H.C., C.C.Y.C., J.-P.C., C.L., C.C.-Y.Y., L.L., 663 K.K.-W.T., H. Chen, H. Chu, J.F.-W.C.

664 Visualization: S.Y., J.F.-W.C.

665 Funding acquisition: G.L., H. Chen, D.-Y.J., K.-Y.Y., J.F.-W.C.

666 Project administration: S.Y., D.-Y.J., K.-Y.Y., J.F.-W.C

667 Supervision: S.Y., D.-Y.J., K.-Y.Y., J.F.-W.C

668 Writing - original draft: S.Y., J.F.-W.C

669 Writing - review \& editing: S.Y., D.-Y.J., K.-Y.Y., J.F.-W.C

670

671 Competing interests: J.F.-W.C. has received travel grants from Pfizer Corporation Hong Kong 672 and Astellas Pharma Hong Kong Corporation Limited and was an invited speaker for Gilead 673 Sciences Hong Kong Limited and Luminex Corporation. K.K.-W.T., H. Chen and K.-Y.Y. report 
674 collaboration with Sinovac Biotech Ltd. and China National Pharmaceutical Group Co., Ltd.

675 (Sinopharm). The other authors declare no competing interests.

676

677 Data availability: Complete sequences of the SARS-CoV-2 Omicron (hCoV-19/Hong 678 Kong/HKU-344/2021; GISAID accession number EPI_ISL_7357684) and Delta (hCoV679 19/Hong Kong/HKU-210804-001/2021; GISAID: EPI_ISL_3221329) variants available through 680 GISAID. Other supporting raw data are available from the corresponding author upon reasonable 681 request. Source data are provided with this paper. 

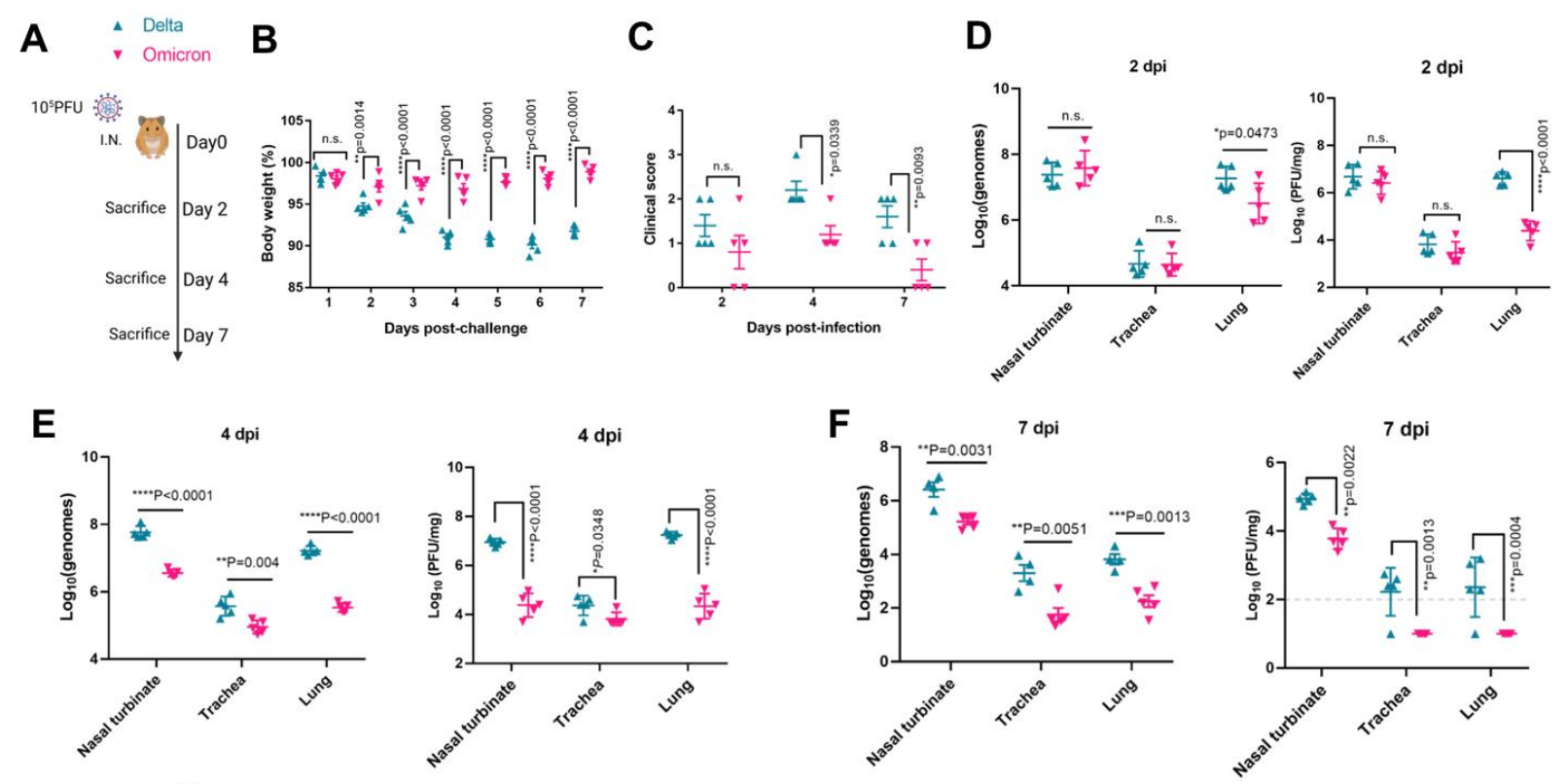

G
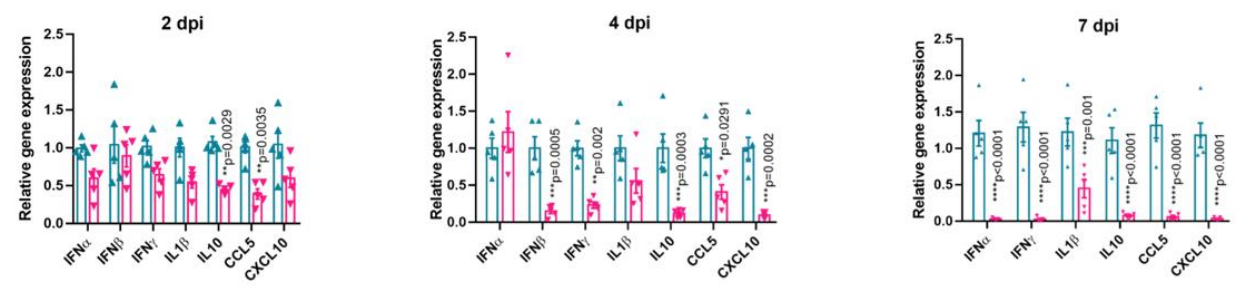

Fig. 1. Pathogenicity of the Omicron and Delta variants in the golden Syrian hamster

684

model. (A) Scheme of the pathogenicity study comparing infections caused by the Omicron and Delta variants in golden Syrian hamsters. At 0 day post-infection (dpi), each hamster was intranasally inoculated with $100 \mu \mathrm{L}$ of DMEM containing $10^{5}$ PFU of SARS-CoV-2 ( $\mathrm{n}=15$ for each variant). The hamsters were sacrificed at $2 \mathrm{dpi}$, $4 \mathrm{dpi}$, and $7 \mathrm{dpi}(\mathrm{n}=5$ per variant per timepoint) for viral load quantitation by qRT-PCR, virus titer quantitation by plaque assay, and histopathological studies. (B) Body weight changes and (C) clinical scores of the hamsters after infection with either variant. A score of 1 was given to each of the following clinical signs: lethargy, ruffled fur, hunchback posture, and rapid breathing. Respiratory tract tissue viral loads (left) and infectious virus titers (right) at (D) 2dpi, (E) 4dpi, and (F) 7dpi (the dotted line represents the limit of detection of the plaque assay at $100 \mathrm{PFU} / \mathrm{mg}$ ). (G) Lung 
bioRxiv preprint doi: https://doi.org/10.1101/2022.01.12.476031; this version posted January 13,2022 . The copyright holder for this preprint (which was not certified by peer review) is the author/funder, who has granted bioRxiv a license to display the preprint in perpetuity. It is made available under aCC-BY-NC-ND 4.0 International license.

694 cytokine/chemokine profiles at 2dpi, 4dpi, and 7dpi. Data are mean \pm standard deviations. $\mathrm{n}=5$

695 biological replicates per variant time-point. $* * * * P<0.0001$, $* * * P<0.001, * * P<0.01$, and $696 * P<0.05$ by two-way ANOVA. 

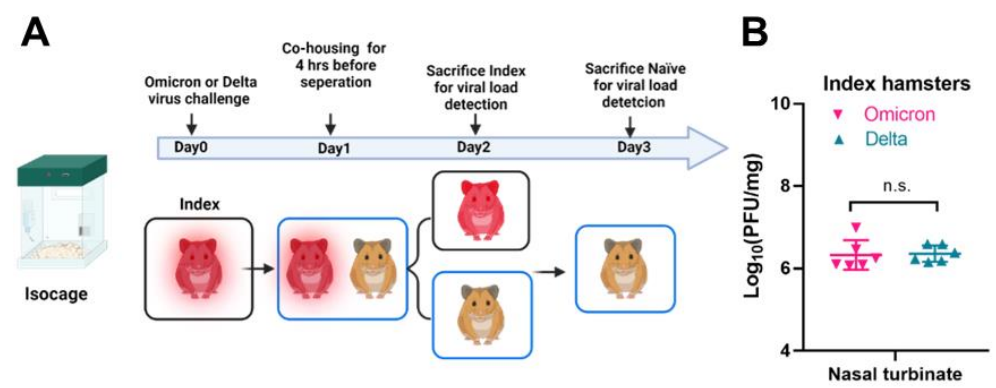

C
\begin{tabular}{|c|c|c|c|}
\hline $\begin{array}{c}\text { Exposed } \\
\text { naïve } \\
\text { hamsters }\end{array}$ & $\begin{array}{c}\text { Omicron } \\
\text { (infected } \\
/ \text { total) }\end{array}$ & $\begin{array}{c}\text { Delta } \\
\text { (infected } \\
/ \text { total) }\end{array}$ & $P$-value \\
\hline Round 1 & $\begin{array}{c}3 / 3 \\
(100 \%)\end{array}$ & $\begin{array}{c}3 / 3 \\
(100 \%)\end{array}$ & 1.000 \\
\hline Round 2 & $\begin{array}{c}3 / 3 \\
(100 \%)\end{array}$ & $\begin{array}{c}3 / 3 \\
(100 \%)\end{array}$ & 1.000 \\
\hline Total & $\begin{array}{c}6 / 6 \\
(100 \%)\end{array}$ & $\begin{array}{c}6 / 6 \\
(100 \%)\end{array}$ & 1.000 \\
\hline
\end{tabular}

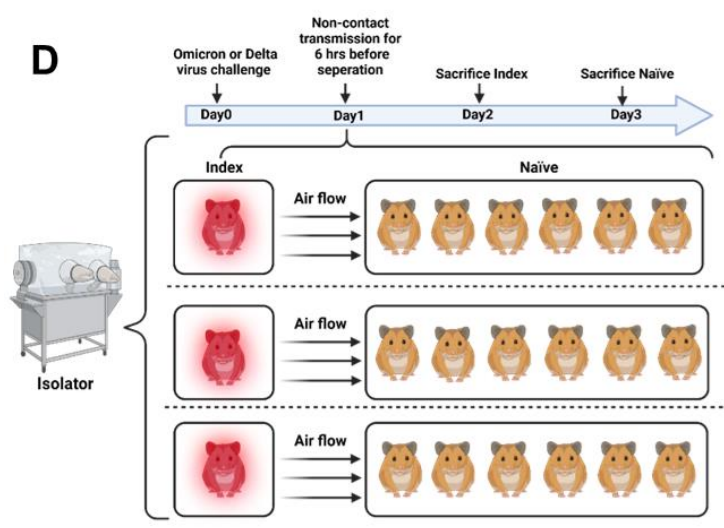

$\mathbf{E}$

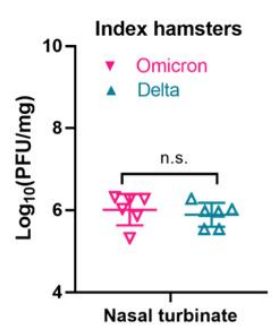

$\mathbf{F}$

\begin{tabular}{|c|c|c|c|}
\hline $\begin{array}{c}\text { Exposed } \\
\text { naïve } \\
\text { hamsters }\end{array}$ & $\begin{array}{c}\text { Omicron } \\
\text { (infected } / \\
\text { total) }\end{array}$ & $\begin{array}{c}\text { Delta } \\
\text { (infected } / \\
\text { total) }\end{array}$ & $\begin{array}{c}P \text { - } \\
\text { value }\end{array}$ \\
\hline Round 1 & $\begin{array}{c}14 / 18 \\
(77.8 \%)\end{array}$ & $\begin{array}{c}11 / 18 \\
(61.1 \%)\end{array}$ & 0.471 \\
\hline Round 2 & $\begin{array}{c}16 / 18 \\
(88.9 \%)\end{array}$ & $\begin{array}{c}13 / 18 \\
(72.2 \%)\end{array}$ & 0.402 \\
\hline Total & $30 / 36$ & $24 / 36$ & 0.173 \\
& $(83.3 \%)$ & $(66.7 \%)$ & \\
\hline
\end{tabular}

Fig. 2. Contact and non-contact transmissions of the Omicron and Delta variants among

golden Syrian hamsters. (A) Scheme of the contact transmission study. (B) The nasal turbinate infectious virus titres in the virus-challenged index hamsters at 2 dpi were determined by plaque assay to ensure successful infection of both groups of hamsters. n.s. indicates non-significant (Student's t-test). (C) Positive rates of infection among the exposed naïve hamsters after exposure to either the Omicron or Delta variant. $P$-values were determined by Chi-square test.

(D) Scheme of the non-contact transmission study. (E) The nasal turbinate infectious virus titres successful infection of both groups of hamsters. n.s. indicates non-significant (Student's t-test). 
A

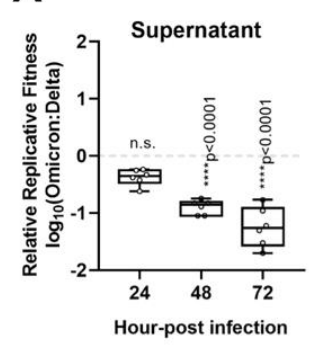

B

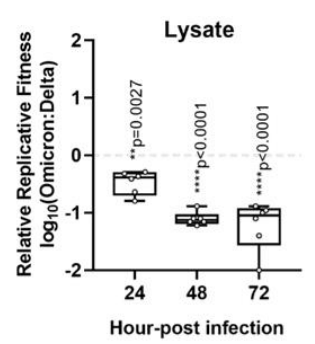

C

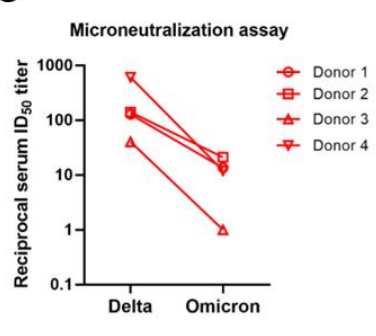

$\mathbf{E}$

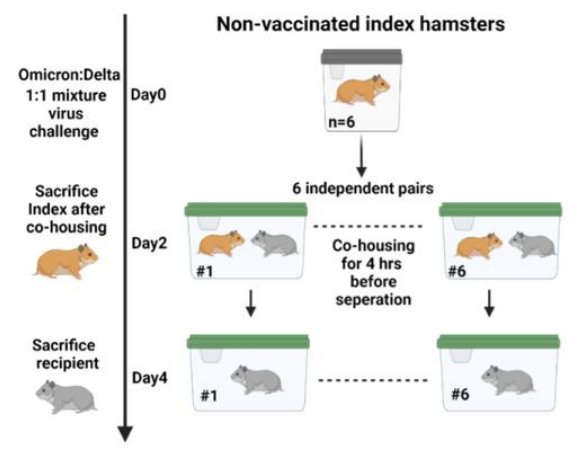

G

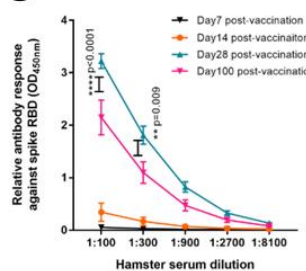

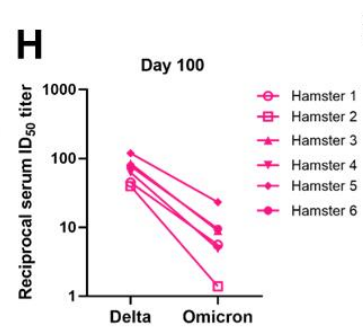

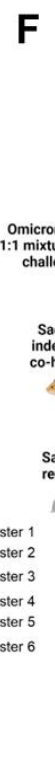

709

710

711

712

713

714

715

716

717

718

719
D

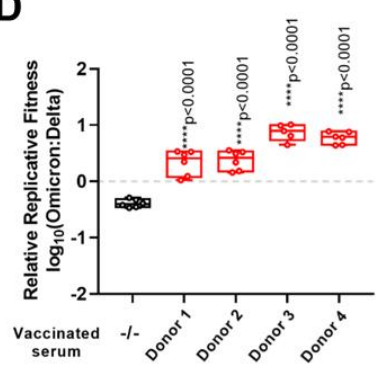

I
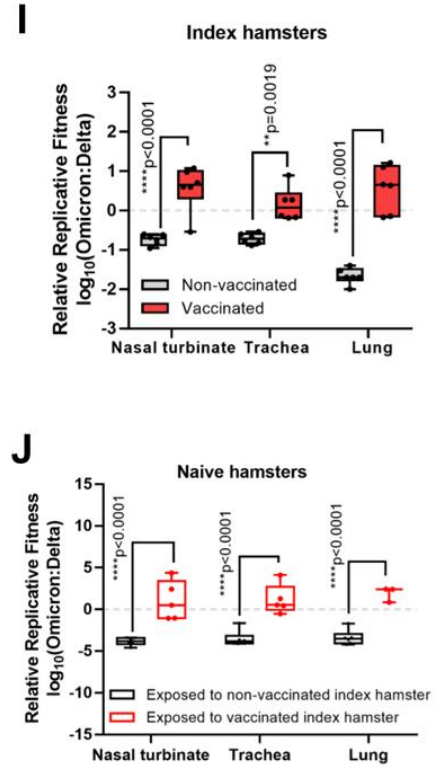

Fig. 3. Comparative in vitro and in vivo fitness of the SARS-CoV-2 Omicron and Delta

variants. (A) In vitro virus competition assay using a mixture of the Omicron and Delta variants with an initial ratio of 1:1 was inoculated onto Calu-3 cell cultures (final MOI of 0.10 for each variant). The ratios after competition in the cell culture (A) supernatant and (B) lysates were measured by qRT-PCR. (C) Serum samples from 4 donors at least 14 days after receiving the second dose of mRNA (Pfizer-BioNTech Comirnaty) vaccine were used to neutralize live Omicron and Delta variants, and the resulting 50\% inhibitory dilution ( ID $\left._{50}\right)$ were displayed. (D) In vitro virus competition with or without addition of the vaccinees' serum samples in cell culture supernatants. Scheme of the in vivo competition models using (E) non-vaccinated and (F) vaccinated index hamsters. (G) The hamster sera $(n=6)$ were collected at the indicated days after 
720 vaccination for detection of antibody response against spike RBD. (H) The neutralizing activity

721 of the hamster serum samples collected at day 100 after vaccination against live Omicron and

722 Delta variants. The Omicron:Delta ratio in the nasal turbinate, trachea, and lung of the (I) non-

723 vaccinated and vaccinated index hamsters, and $(\mathbf{J})$ naïve hamsters exposed to the non-vaccinated

724 or vaccinated index hamsters. Data are mean \pm standard deviations. $* * * * P<0.0001, * * * P<0.001$,

$725 * * P<0.01$, and $* P<0.05$ by two-way ANOVA. 
A

Oral swab

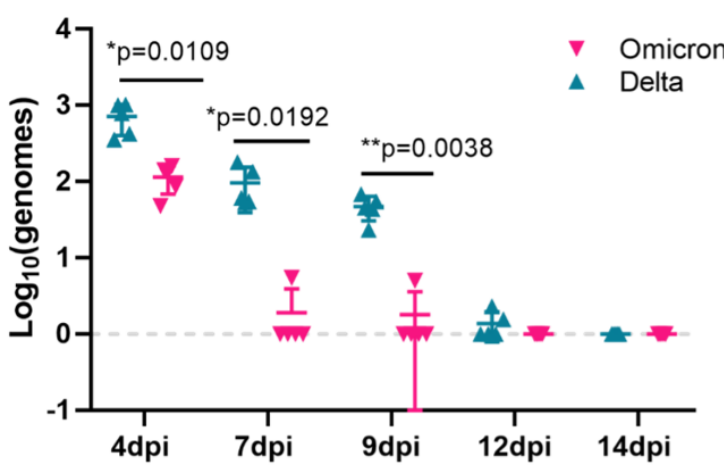

B

Feces

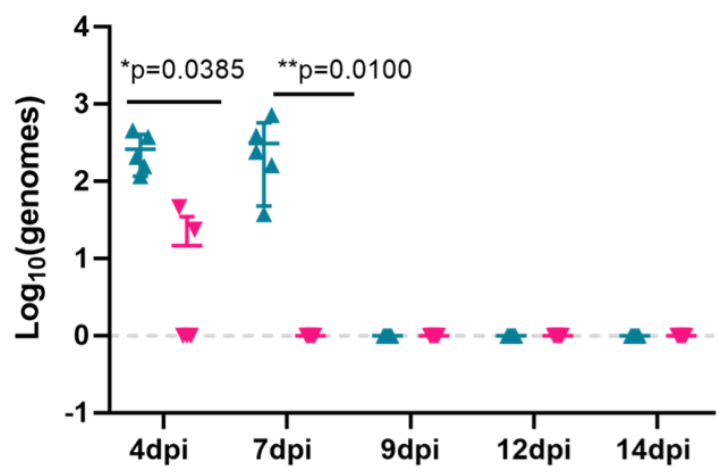

727 Fig. S1. Virus shedding in the oral swabs and feces of the Omicron-infected and Delta-

728 infected hamsters. (A) Oral swabs and (B) feces were serially collected from the Omicron-

729 infected and Delta-infected hamsters at the indicated time-points for viral load detection by

730 quantitative reverse transcription-polymerase chain reaction. The dotted line represented the

731 limit of detection. Data are mean \pm standard deviations. $n=5$ biological replicates per variant.

$732 * * P<0.01$ and $* P<0.05$ by two-way ANOVA. 


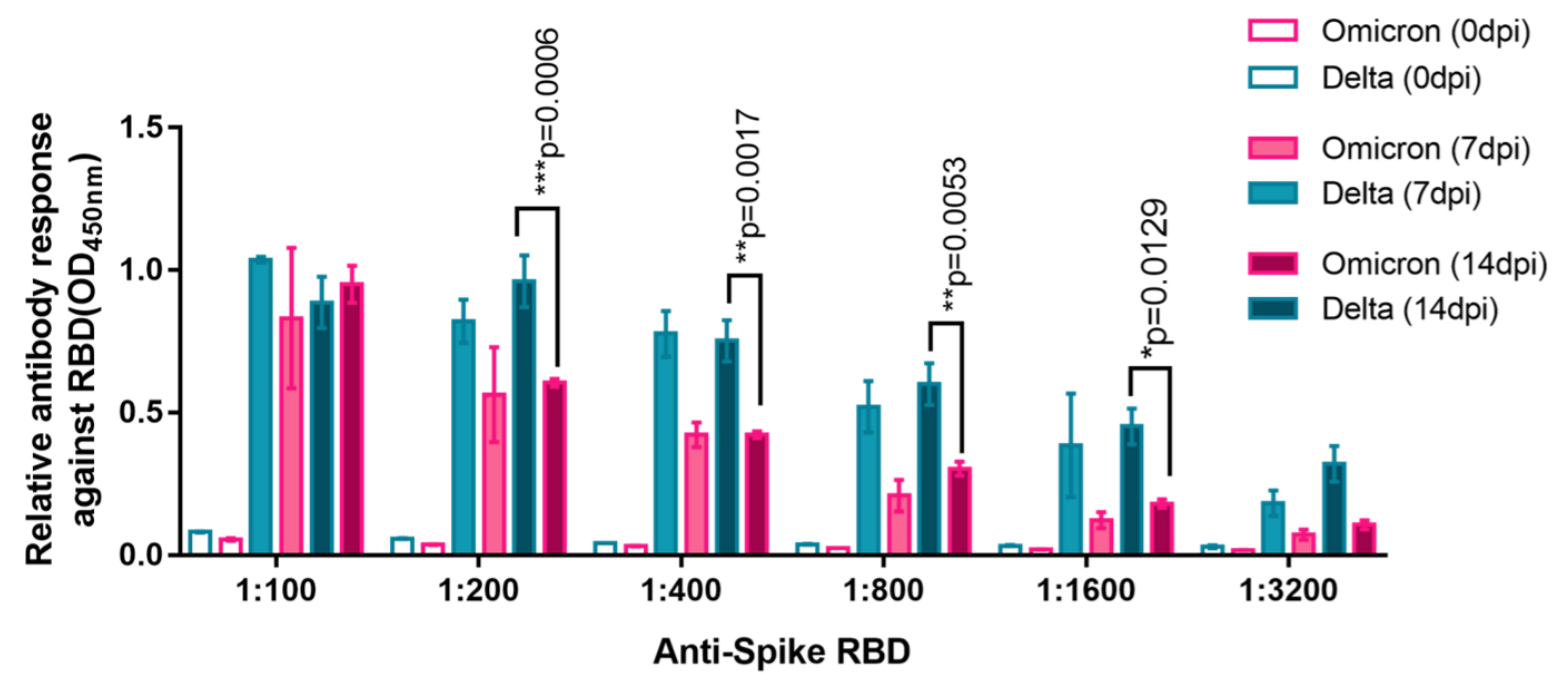

734 Fig. S2. Variant-specific anti-spike receptor-binding domain antibody response of the

Omicron-infected and Delta-infected hamsters. Microwell plates were coated with 100 ng/well of SARS-CoV-2 RBD specific for each variant (Omicron RBD, SinoBiological

737 \#Cat:40592-V08H121 and Delta RBD, Sinobiological Cat\# Cat:40592-V08H90) overnight at

$7384^{\circ} \mathrm{C}$, followed by incubation with blocking reagent overnight at $4{ }^{\circ} \mathrm{C}$. After removal of the 739 blocking solution, aliquots of $100 \mu \mathrm{l} /$ well of serially diluted hamster sera from the dilution of $740 \quad 1: 100$ were added to microwell plates coated with RBD protein and incubated at $37^{\circ} \mathrm{C}$ for $1 \mathrm{~h}$.

741 The plates were then washed 6 times, rabbit anti-hamster horseradish peroxidase antibody (100 $742 \mu \mathrm{L} /$ well) at the dilution of 1:2000 was added and incubated for $30 \mathrm{~min}$ at $37^{\circ} \mathrm{C}$. After the plates 743 were washed 6 times, tetramethylbenzidine substrate $(100 \mu \mathrm{L} /$ well $)$ was added. The reaction was 744 stopped after 10 min by the addition of $0.3 \mathrm{~N}$ sulfuric acid, and the plates were then examined in 745 an ELISA plate at $450 \mathrm{~nm}$ and $620 \mathrm{~nm} . \mathrm{n}=5$ biological replicates per variant. $* * * * P<0.0001$, $* * * P<0.001, * * P<0.01$, and $* P<0.05$ by two-way ANOVA. 


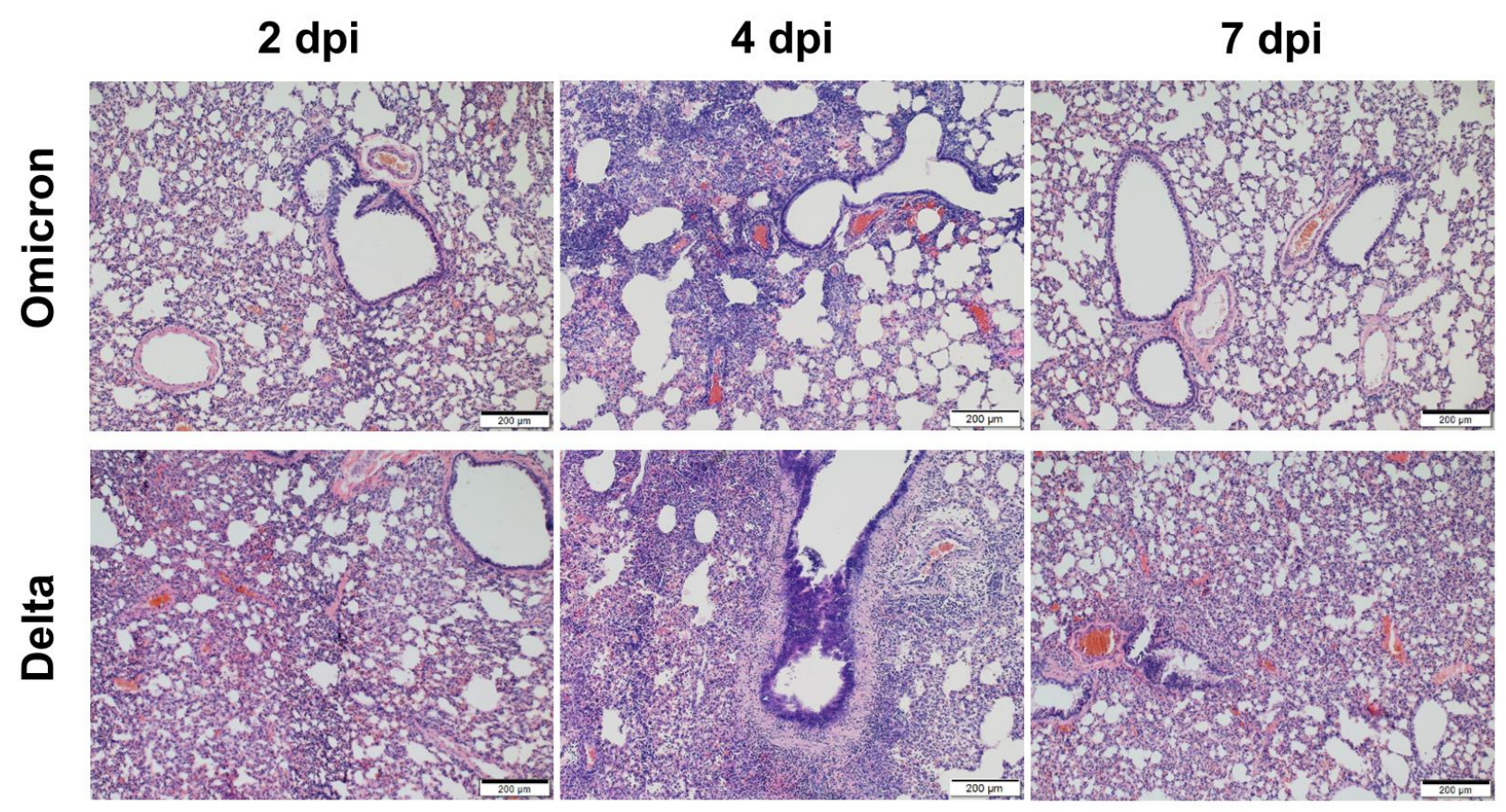

Fig. S3. Representative histopathological findings of the lung of Omicron-infected and

Delta-infected hamsters. Representative haematoxylin and eosin-stained lung sections of the

Omicron-infected and Delta-infected hamsters were collected at 2 days post-infection (dpi), 4

$751 \mathrm{dpi}$, and $7 \mathrm{dpi}$. At 2 dpi and $4 \mathrm{dpi}$, the Delta-infected hamsters showed more diffuse

752 peribronchiolar, perivascular, and alveolar inflammatory infiltrates, bronchiolar epithelial

753 destruction, and alveolar congestion than the Omicron-infected hamsters. At 7 dpi, the

754 histopathological changes have mostly resolved in the lungs of the Omicron-infected hamsters,

755 while the Delta-infected hamsters still demonstrated blood vessel congestion and alveolar wall

756 inflammatory infiltration. Scale bars $=200 \mu \mathrm{M}$. 
A 2 dpi 4 dpi
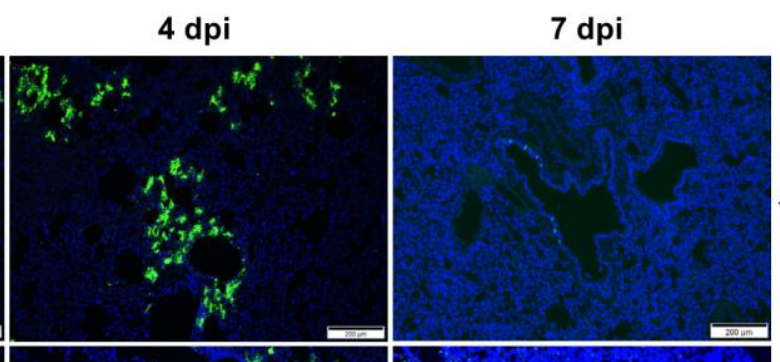

B
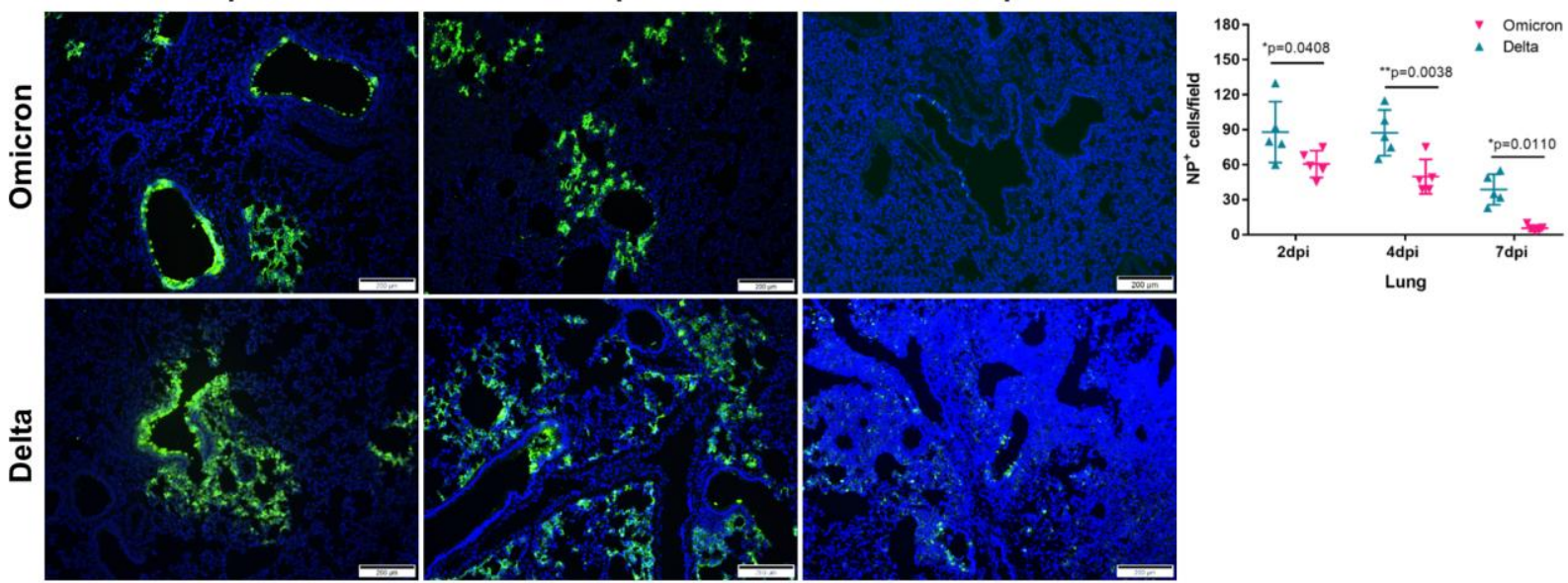

Fig. S4. Representative immunofluorescence staining findings of the lung of Omicron-

759 infected and Delta-infected hamsters. (A) Immunofluorescence staining of the lung sections of

760 Omicron-infected and Delta-infected hamsters at 2 days-post infection (dpi), 4 dpi, and 7 dpi.

761 SARS-CoV-2 nucleocapsid (NP, green) and cell nuclei (blue) were stained. These representative

762 images were selected from a pool of 15 images captured in 5 hamsters per variant. (B) NP-

763 positive cells per $50 \times$ field per lung section of each hamster. Scale bars $=200 \mu \mathrm{m}$. Data are mean

$764 \pm$ standard deviations. $\mathrm{n}=5$ biological replicates per variant. $* * P<0.01$ and $* P<0.05$ by two-way

765 ANOVA. 


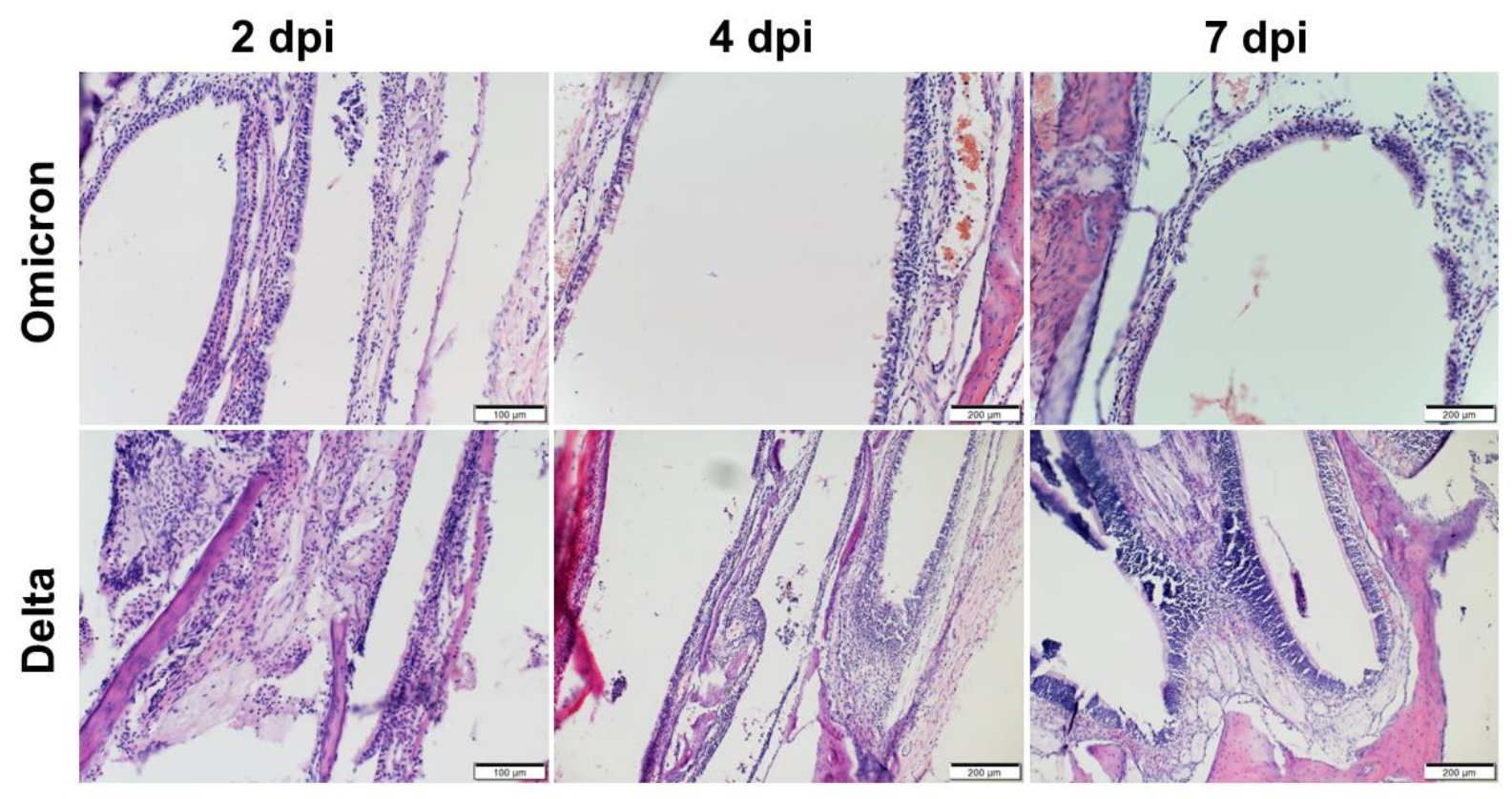

Fig. S5. Representative histopathological findings of the nasal turbinate of Omicroninfected and Delta-infected hamsters. Representative haematoxylin and eosin-stained lung sections of the Omicron-infected and Delta-infected hamsters were collected at 2 days postinfection (dpi), $4 \mathrm{dpi}$, and $7 \mathrm{dpi}$. At $2 \mathrm{dpi}$, submucosal infiltration and destruction of the

771 epithelium were observed in both groups of hamsters. At 4 dpi, the Delta-infected hamsters

772 showed severe destructive changes of the respiratory and olfactory epithelia, while the Omicron-

773 infected hamsters showed intact respiratory epithelium with mild intra-epithelial inflammatory

774 infiltrates. At 7 dpi, the histopathological changes have mostly resolved in the Omicron-infected

775 hamsters, while the Delta-infected hamsters still demonstrated some residual inflammation. Scale

$776 \quad$ bars $=100 \mu \mathrm{M}$ or $200 \mu \mathrm{M}$. 
A
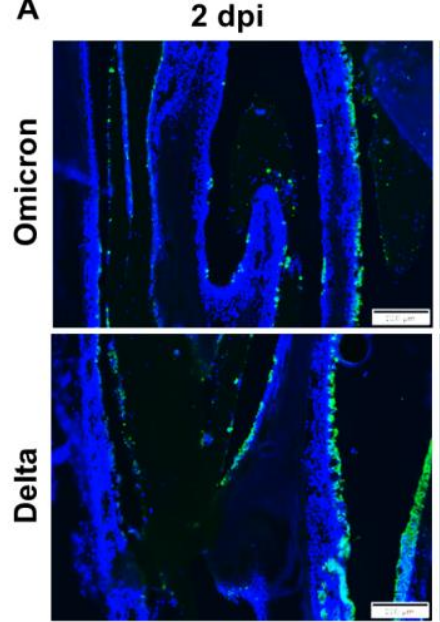

$4 \mathrm{dpi}$

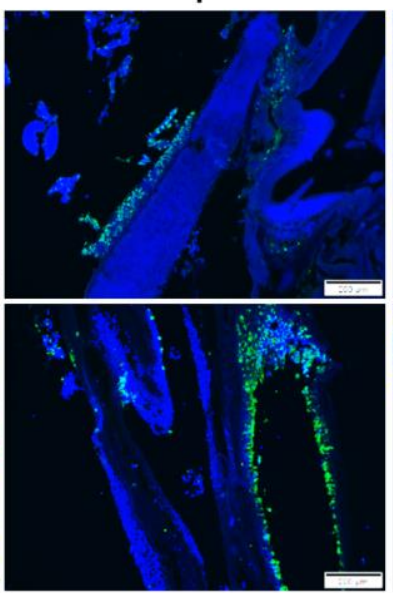

$7 \mathrm{dpi}$

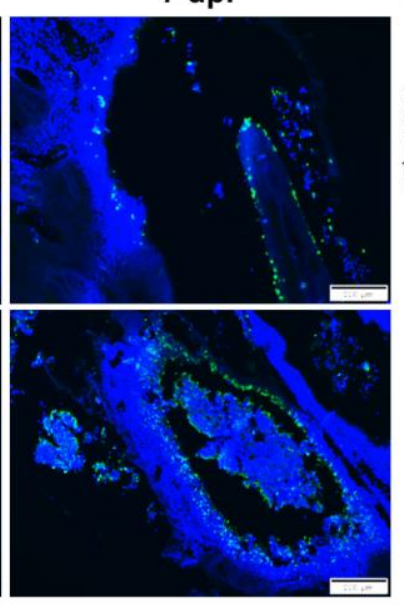

B

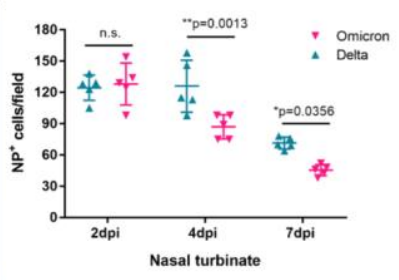

Fig. S6. Representative immunofluorescence staining findings of the nasal turbinate of

779 Omicron-infected and Delta-infected hamsters. (A) Immunofluorescence staining of the nasal

780 turbinate sections of Omicron-infected and Delta-infected hamsters at 2 days-post infection (dpi),

7814 dpi, and 7 dpi. SARS-CoV-2 nucleocapsid (NP, green) and cell nuclei (blue) were stained.

782 These representative images were selected from a pool of 15 images captured in 5 hamsters per

783 variant. (B) NP-positive cells per 50× field per nasal turbinate section of each hamster. Scale

784 bars $=200 \mu \mathrm{m}$. Data are mean \pm standard deviations. $\mathrm{n}=5$ biological replicates per variant.

$* * P<0.01$ and $* P<0.05$ by two-way ANOVA. 
A

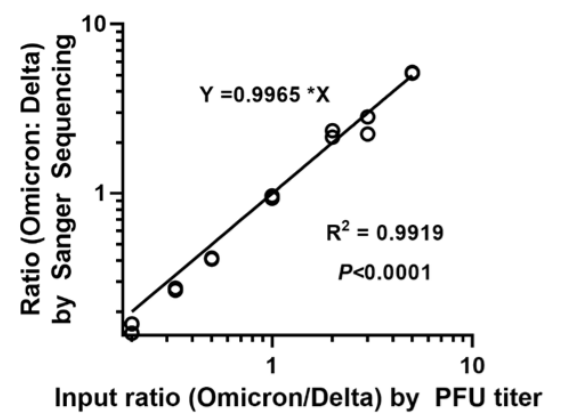

B

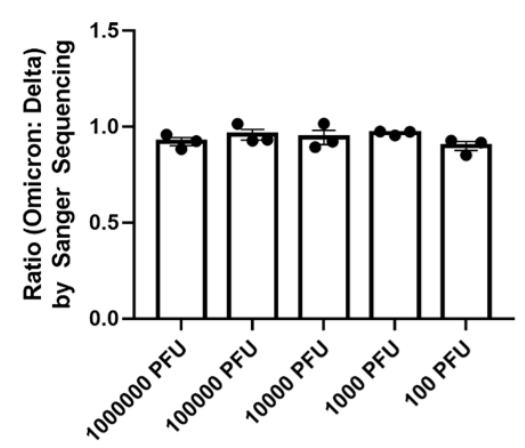

C

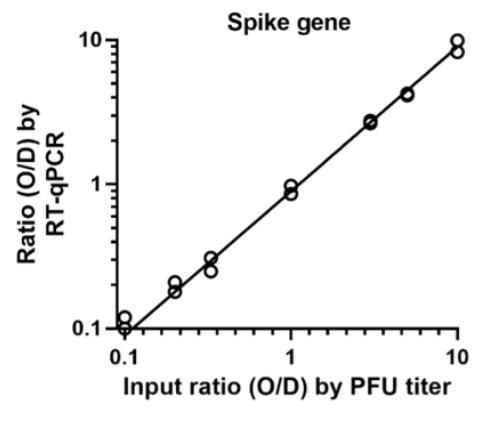

Fig. S7. Validation of the competition assay. (A) The correlation between input PFU ratios and output RT-PCR amplicon ratios determined by Sanger sequencing, i.e. R493 for Omicron (CGA) and Q493 for Delta (CAA). The Omicron and Delta variants were mixed at PFU ratios of 10:1, $5: 1,3: 1,1: 1,1: 3,1: 5$, or $1: 10$. Total RNA of the mixture was extracted and amplified by RTPCR. The R493/Q493 ratio was calculated by the peak heights of Sanger sequencing. Data were analyzed by linear regression with correlation coefficients (r) and significance (p). The symbols represent individual replicates and the solid line represents the fitted line. Data were derived

794 from an experiment conducted in duplicate. (B) Assay range evaluation. The ratio of 795 Omicron:Delta variant mixture determined by Sanger sequencing was consistent in a wide range of virus amounts. The two variants were mixed at 1:1 ratio. The total titers of the mixed virus 797 stock were $10^{2}, 10^{3}, 10^{4}, 10^{5}$, and $10^{6} \mathrm{PFU}$. The total RNA of the virus mixture was isolated and amplified by RT-PCR. The R493/Q493 ratio was calculated by the peak heights from Sanger sequencing. The symbols represent individual replicates, bar heights represent the means, and error bars represent standard deviations. Data are derived from an experiment conducted in the Omicron and Delta variants. 